\title{
Hidrólise ácida de jornal para a produção de açúcares fermentescíveis e energia
}

\section{Production of fermentable sugars and energy from acid hydrolysis of newspaper}

\author{
${ }^{1}$ Bruno Chaboli Gambarato \\ 2 Sarah Evellin Silva de Moraes \\ ${ }^{2}$ Nayra Cardoso Gomes \\ ${ }^{3}$ Bruno Silva Dantas \\ ${ }^{4}$ Anderson Luiz do Carmo
}

\footnotetext{
1 Docente do Centro Universitário de Volta Redonda - UniFOA. bruno.gambarato@foa.org.br

2 Discente do Curso de Engenharia Ambiental, Centro Universitário de Volta Redonda - UniFOA.

3 Discente do Curso de Engenharia Civil, Centro Universitário de Volta Redonda - UniFOA.

4 Engenheiro Bioquímico (USP) e Discente do Curso de Engenharia Industrial Madeireira (UFPR).
}

\section{Resumo}

Neste trabalho, foi realizada a hidrólise ácida de papel jornal com o objetivo de produzir açúcares fermentescíveis, principalmente glicose e xilose. As reações de hidrólise se processaram a $121^{\circ} \mathrm{C}$, utilizando $\mathrm{H}_{2} \mathrm{SO}_{4} 2,59 \% \mathrm{~m} / \mathrm{m}$ nos tempos de 15 a 120 min. Verificou-se que a hidrólise dos polissacarídeos ocorre segundo uma reação de pseudoprimeira ordem com constante de velocidade $\mathrm{k}=0,0168 \mathrm{~min}^{-1}$ e que, em 60 min, a concentração de açúcares no hidrolisado atinge o seu valor ótimo. Após esse tempo, ocorrem reações de formação de compostos de desidratação, que atuam como inibidores de fermentação e são, portanto, inviáveis ao processo. Foi verificado ainda, por Espectrometria de Infravermelho e por Análise Elementar $\mathrm{CHNS/O}$, que o resíduo sólido da hidrólise é, principalmente, lignina e polissacarídeos não hidrolisados e apresenta poder calorífico superior de $23 \mathrm{~kJ} \mathrm{~g}^{-1}$. Por fim, verificou-se que a partir de $1 \mathrm{~kg}$ de jornal, podem ser produzidos 341,3 g de açúcares fermentescíveis e 4,51 MJ de energia.

\section{Palavras-chave}

Papel jornal; hidrólise ácida; biomassa; açúcares fermentescíveis.

\begin{abstract}
In this paper, waste newspaper acid hydrolysis was performed in order to produce fermentable sugars, especially glucose and xylose. Hydrolysis reactions were processed at $121^{\circ} \mathrm{C}$ using $2.59 \% \mathrm{~W} / \mathrm{W} \mathrm{H}_{2} \mathrm{SO}_{4}$ for $15-120 \mathrm{~min}$. It was found that hydrolysis of polysaccharides occurs according to a pseudo first order reaction rate constant $k=0.0168 \mathrm{~min}^{-1}$ and in $60 \mathrm{~min}$, the concentration of sugars in hydrolyzates reaches its optimal value. After this time, dehydration reactions occur forming compounds, which act as fermentation inhibitors and are therefore impractical for the process. It was further verified by infrared spectroscopy and by elemental analysis CHNS/O that the hydrolysis solid residue, which is mainly lignin and unhydrolysed polysaccharides, has a heat power of $23 \mathrm{~kJ} \mathrm{~g}^{-1}$. Finally, it was found that from $1 \mathrm{~kg}$ of waste newspaper can be produced $341.3 \mathrm{~g}$ of fermentable sugars and $4.51 \mathrm{MJ}$ of energy.
\end{abstract}

\section{Keywords}

Waste newspaper; acid hydrolysis; biomass; fermentable sugars.

\section{Como você deve citar?}

GAMBARATO, Bruno Chaboli et al. Hidrólise ácida de jornal para a produção de açúcares fermentescíveis e energia. Cadernos UniFOA, Volta Redonda, n. 27, p. 25-32, abr. 2015. 


\section{INTRODUÇÃO}

Os jornais estão entre os principais meios de comunicação e de publicidade no Brasil. Atualmente, o consumo brasileiro de papel jornal é de cerca de 540.000 toneladas por ano, sendo que a produção local é 128.000 toneladas e o restante é importado (BRACELPA, 2014). Os dados mais recentes mostram que o Brasil é, hoje, $04^{\circ}$ produtor mundial de celulose e o $11^{\circ}$ produtor de papel e papelão (BRACELPA, 2014).

O papel jornal é produzido pela mistura de pasta mecânica com alguma quantidade de polpa química de fibra longa para dar resistência à folha úmida do papel. A essa mistura, são adicionadas, ainda, parcelas significativas de polpas recicladas. Assim, a composição aproximada de um papel jornal consiste de 70 a $85 \%$ de pasta de alto rendimento, podendo ser pasta mecânica ou termomecânica, 10 a $15 \%$ de polpa química semibranqueada ou branqueada de fibra longa e 5 a $20 \%$ de fibras secundárias recicladas (EK et al., 2009).

A madeira utilizada como matéria-prima para a produção de pasta celulósica provém de várias espécies arbóreas de Eucalyptus e Pinus. A madeira, que é um material lignocelulósico, é composta por celulose, hemiceluloses, lignina, cinzas inorgânicas e extrativos (HON; SHIRAISHI, 2001).

A conversão de jornal a etanol é um processo que compreende diversas etapas. A principal delas é a hidrólise, que converte os carboidratos presentes no papel (fração de celulose e hemiceluloses, denominada Holocelulose) em açúcares fermentescíveis, principalmente glicose e xilose, que podem ser convertidos, via fermentação, a etanol e outros produtos de maior valor agregado. As reações de hidrólise podem ocorrer tanto por via química, quanto por via enzimática. Uma vez que os insumos requeridos para a hidrólise enzimática ainda são caros, este trabalho objetiva estudar a hidrólise ácida do jornal para produção de açúcares fermentescíveis, utilizando um composto químico barato e aplicável na indústria: o ácido sulfúrico.

Por meio de hidrólise ácida, é possível hidrolisar quase que totalmente a celulose e a hemicelulose presentes no material lignocelulósico. Entretanto, podem ocorrer reações de desidratação, formando furfural e hidroximetilfurfural, conhecidos compostos inibidores de fermentação (RAMOS, 2003). Sendo assim, torna-se necessário um estudo dessas reações, visando encontrar uma condição de processo que gere elevadas quantidades de açúcares fermentescíveis, com baixa produção de inibidores, que é o objetivo deste trabalho.

\section{MATERIAIS E MÉTODOS}

\subsection{Caracterização do jornal}

0 teor de holocelulose foi determinado por meio da reação do jornal livre de extrativos com clorito de sódio $5 \% \mathrm{~m} / \mathrm{m}$ e ácido acético $5 \% \mathrm{v} / \mathrm{v}$, a $70^{\circ} \mathrm{C}$ por 5 horas. Em seguida, o sistema foi resfriado para $25^{\circ} \mathrm{C}$, filtrado, lavado com água até pH neutro, seco e pesado em balança analítica. Para a determinação do teor de lignina, procedeu-se a hidrólise de $1,0 \mathrm{~g}$ de jornal seco com $\mathrm{H}_{2} \mathrm{SO}_{4} 72 \% \mathrm{~m} / \mathrm{m}$, a $25^{\circ} \mathrm{C}$ por $2 \mathrm{~h}$, seguida de diluição para $2,5 \% \mathrm{~m} / \mathrm{m}$ de ácido, autoclavada a $121^{\circ} \mathrm{C}$ por $30 \mathrm{~min}$. 0 material foi filtrado e o resíduo foi lavado exaustivamente com água, seco e pesado em balança analítica. 0 teor de cinzas no material e na lignina foi determinado após calcinação dos respectivos materiais a $800^{\circ}$ $\mathrm{C}$ por $2 \mathrm{~h}$, em forno tipo mufla. 


\subsection{Hidrólise Ácida}

As reações de hidrólise se processaram empregando-se $10 \mathrm{~g}$ de jornal seco em um meio contendo $\mathrm{H}_{2} \mathrm{SO}_{4} 2,59 \% \mathrm{~m} / \mathrm{m}$ a $121^{\circ} \mathrm{C}$, nos tempos de 15 a 120 minutos, com relação sólido:líquido 1:10. 0 sistema reacional foi, então, resfriado à temperatura ambiente e a mistura foi filtrada. Os hidrolisados foram armazenados em geladeira e o resíduo foi seco e encaminhado para análise.

\subsection{Caracterização dos hidrolisados}

Os hidrolisados foram caracterizados quanto ao teor de açúcares redutores, a partir da reação de $1,0 \mathrm{~mL}$ de amostra com $1,0 \mathrm{~mL}$ do reagente de DNS (ácido 3,4 dinitrossalicílico), por $5 \mathrm{~min}$ a $100^{\circ}$ C. Ao término da reação, foram adicionados $13,0 \mathrm{~mL}$ de água deionizada e foi realizada a leitura de absorbância a $540 \mathrm{~nm}$ em espectrofotômetro. As concentrações de açúcares redutores foram determinadas a partir de uma curva de calibração feita com soluções padrão de glicose nas mesmas condições acima. A absorbância relativa a $280 \mathrm{~nm}$, relacionada à formação dos compostos inibidores de fermentação, foi determinada a partir da diluição de $5 \mathrm{~mL}$ de hidrolisado e cerca de $2 \mathrm{~mL}$ de $\mathrm{NaOH} 6,5$ $\mathrm{N}$ em balão volumétrico de $100,0 \mathrm{~mL}$ com água deionizada. Após a diluição, foi feita a leitura de absorbância do hidrolisado a $280 \mathrm{~nm}$ e o valor obtido foi multiplicado pelo fator de diluição para obtenção da absorbância relativa.

\subsection{Caracterização do resíduo}

A determinação dos teores de carbono, hidrogênio, nitrogênio e enxofre no resíduo da hidrólise foi realizada em equipamento Perkim Elmer PE 2400 série II CHNS/O, no Laboratório Associado de Combustão e Propulsão do Instituto Nacional de Pesquisas Espaciais - INPE, de Cachoeira Paulista - SP. 0 teor de oxigênio foi determinado por diferença. 0 resíduo da hidrólise foi, ainda, caracterizado por Espectrometria no Infravermelho em um equipamento Spectrum GX Perkin Elmer.

\section{RESULTADOS E DISCUSSÃO}

\subsection{Caracterização do jornal}

A caracterização do jornal quanto aos teores de holocelulose, lignina e cinzas é mostrada na Tabela 1. Para comparação, a tabela também exibe a composição química de outras biomassas vegetais importantes.

Tabela 1 - Composição do jornal e outras biomassas vegetais

\begin{tabular}{cccccc}
\hline Material & Holocelulose & Lignina & Cinzas & Total & Referência \\
\hline Jornal & $74,41 \pm 1,33$ & $19,56 \pm 0,61$ & $3,83 \pm 0,25$ & 97,80 & Este trabalho \\
\hline Jornal & 74,72 & 18,37 & 6,88 & 99,97 & Wu; Huang; Shih, 2014 \\
\hline Palha de cana & $71,41 \pm 1,12$ & $24,98 \pm 0,50$ & $3,19 \pm 0,12$ & 99,58 & Gambarato, 2014 \\
\hline Bagaço de cana & 75,08 & 24,12 & 1,17 & 100,37 & Maziero, 2013 \\
\hline Palha de milho & 59,0 & 21,2 & 6,5 & 86,70 & Öhren et al., 2007 \\
\hline Palha de trigo & 77,70 & 17,0 & 1,80 & 96,50 & Xu et al, 2006 \\
\hline
\end{tabular}


Percebe-se que o jornal apresenta em sua composição um teor de $74,41 \%$ de polissacarídeos (holocelulose), que podem ser convertidos, via hidrólise ácida, a monômeros de açúcares fermentescíveis. Esses açúcares podem ser fermentados para gerar etanol e outros produtos. Quanto à lignina, o jornal se mostra com um teor menor que a biomassa da cana, isso porque, na produção de papel jornal são utilizadas matérias-primas que sofreram processos prévios de deslignificação branda (polpação de alto rendimento) e intensa (polpação química). Dentre os materiais da tabela 1, a palha de trigo é aquela que apresenta os maiores teores de polissacarídeos e o menor teor de lignina. Quantos às cinzas inorgânicas, o jornal é o material com o maior conteúdo dessas substâncias. A presença de altos teores de cinzas promove a corrosão em caldeiras, fato que contribui para a inviabilidade da aplicação direta dessa biomassa na conversão direta para energia.

\subsection{Caracterização dos hidrolisados e cinética de hidrólise}

Os hidrolisados foram caracterizados quanto ao teor de açúcares redutores e à absorbância relativa a $280 \mathrm{~nm}$, como mostram as Figuras 1 e 2. A hidrólise ácida foi capaz de produzir, depois de 60 min, um hidrolisado contendo 38,76 g/L de açúcares fermentescíveis. Após 120 min de reação, o teor de açúcares redutores foi de $41,19 \mathrm{~g} / \mathrm{L}$, o que não representa um aumento considerável na eficiência de hidrólise em relação ao tempo de $60 \mathrm{~min}$. Esse comportamento é explicado pela incidência das reações de formação de compostos de desidratação de monossacarídeos, como furfural e hidroximetilfurfural, que são produzidos a partir da desidratação de pentoses e hexoses, respectivamente. Esses compostos são conhecidos inibidores de fermentação e necessitam ser removidos do meio fermentativo numa etapa posterior à hidrólise, também chamada de etapa de destoxificação do hidrolisado.

Figura 1 - Concentração de açúcares redutores nos hidrolisados

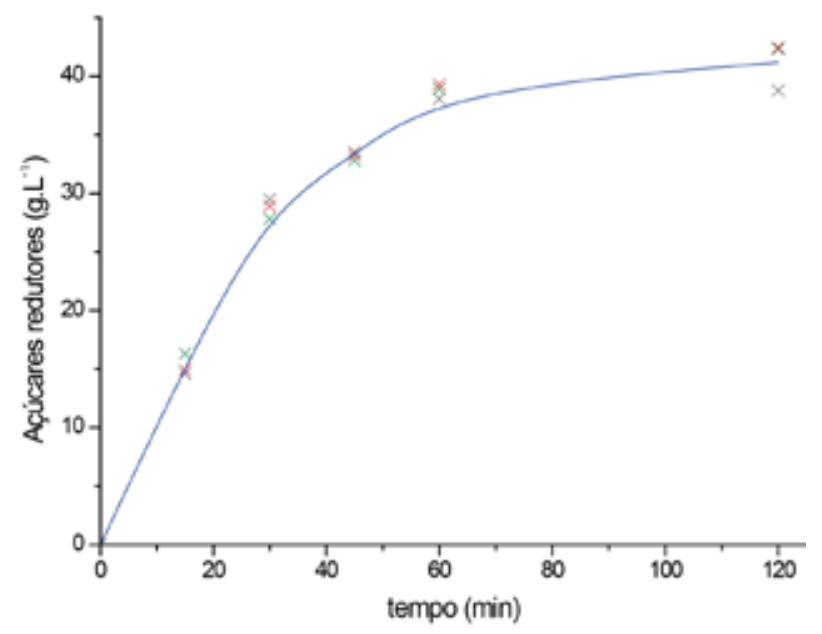

Fonte: dos autores, 2015.

Além do furfural e hidroximetilfurfural, que, em meio ácido, podem ainda dar origem a furano, ácido levulínico e ácido 2-furóico, outros compostos inibidores podem ser formados durante a hidrólise. A lignina sofre reações em meio ácido e é, inicialmente, degradada por meio da clivagem homolítica da ligação $\beta-0-4$ e outras ligações ácido-lábeis (RAMOS, 2003). A incidência dessas reações aumenta com a severidade do processo, gerando compostos fenólicos, que, junto com os furanos, absorvem luz ultravioleta em torno de $280 \mathrm{~nm}$. Assim, a formação desses subprodutos indesejados pode ser verificada por meio da leitura da absorbância relativa do hidrolisado a $280 \mathrm{~nm}$, como mostra a figura 2. 
Figura 2 - Absorbância relativa (280 nm) dos hidrolisados

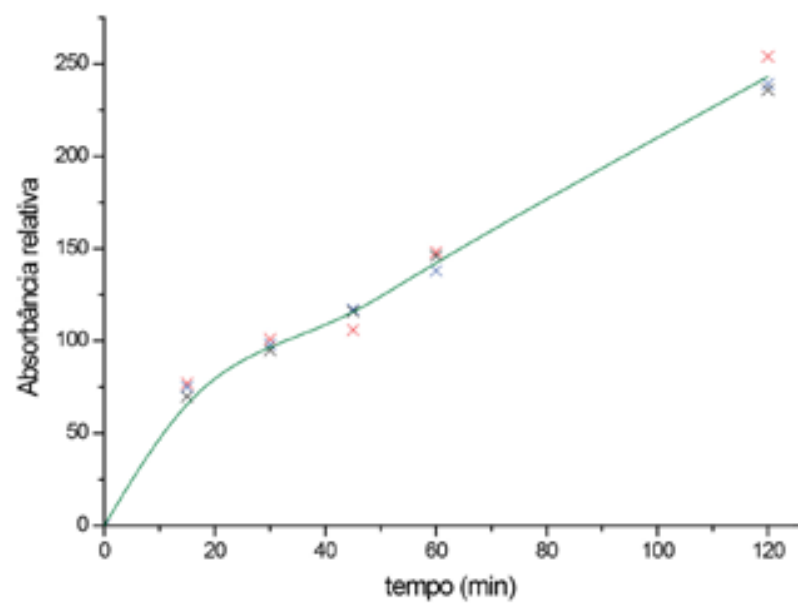

Fonte: dos autores, 2015.

A absorbância relativa aumenta ao longo de toda a reação e, de 60 a 120 min, ocorre um crescimento considerável nessa variável sem consequente formação de açúcares, fato que inviabiliza o tempo de hidrólise de $120 \mathrm{~min}$. Assim, dentro das condições estudadas, o tempo ótimo para a hidrólise é o de $60 \mathrm{~min}$. Para estudar a cinética da hidrólise ácida, o teor de holocelulose no resíduo sólido foi determinado para cada tempo de reação, como mostra a Figura 3.

Verifica-se na Figura 3 que, após 60 min de reação, o resíduo sólido apresenta $34 \%$ de holocelulose, o que representa uma eficiência de hidrólise de $54 \%$. Após $120 \mathrm{~min}$, o teor de polissacarídeos no resíduo sólido atinge valores próximos a $18 \%$, com eficiência de hidrólise de $75 \%$. Entretanto, como mostrado na Figura 1, não há produção considerável de açúcares redutores entre 60 e 120 min, o que sugere, junto com o aumento na absorbância relativa, que, nessa etapa, a formação dos compostos de desidratação de monossacarídeos ocorre em concomitância à formação dos açúcares redutores. Tal fato corrobora a escolha do tempo de 60 min como tempo ótimo para a produção de açúcares fermentescíveis.

Figura 3 - Teor de holocelulose no material ao longo da hidrólise ácida

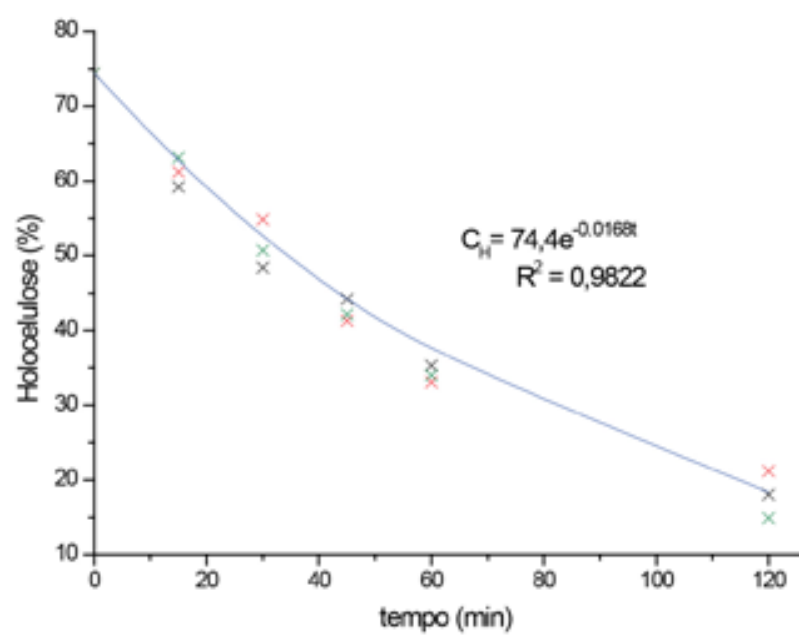

Fonte: dos autores, 2015. 
A cinética de hidrólise pode ser avaliada, considerando-se que o teor de holocelulose no jornal varia com o tempo segundo uma reação de pseudoprimeira ordem, representada pela Equação (1):

$$
-\frac{d C_{H}}{d t}=k C_{H}
$$

Onde é a concentração (\%) de holocelulose no sólido, é o tempo de reação e é a constante de velocidade. Rearranjando a Equação (1) e, introduzindo-se os limites de integração, obtém-se a Equação (2):

$$
\int_{C_{0}}^{C_{H}} \frac{d C_{H}}{C_{H}}=k \int_{0}^{t} d t
$$

Onde representa o teor de holocelulose no jornal antes da hidrólise (74,4\%). A Equação (2) inte-
g r a d a
resu I t a
no
$\mathrm{m}$ o d e I o
d a
E q u a ç ã o
( 3 ).

$$
C_{H}=C_{0} e^{-k t}
$$

O ajuste da Equação (3) aos dados experimentais, realizado pela implementação do método dos mínimos quadrados em linguagem Scilab, é exibido na figura 3. Do ajuste, tem-se que a constante de velocidade da reação apresenta valor $\mathrm{k}=0,0114 \mathrm{~min}^{-1}$, com coeficiente de correlação $\mathrm{R}^{2}=0,9822$.

\subsection{Caracterização do resíduo da hidrólise}

O resíduo sólido da hidrólise ácida por 60 min foi caracterizado por Espectrometria de Infravermelho com Transformada de Fourier (FTIR) e por análise elementar CHNS/O.

Figura 4 - Espectro de Infravermelho com Transformada de Fourier (FTIR) do resíduo da hidrólise ácida de jornal

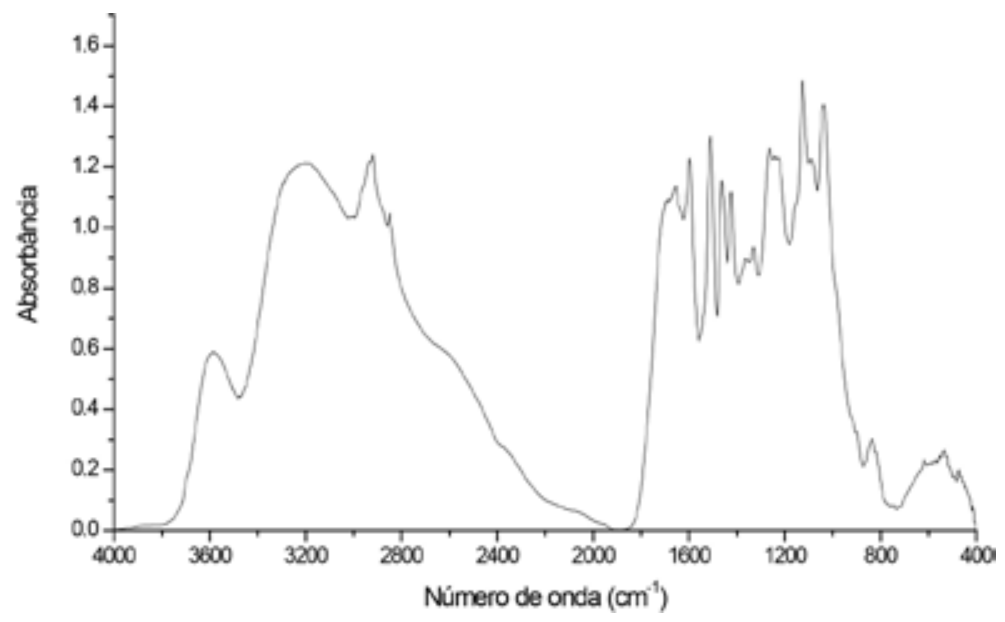

Fonte: dos autores, 2015.

Verifica-se no espectro de infravermelho, mostrado na Figura 4, uma banda de absorção em 1700 $\mathrm{cm}^{-1}$, correspondente ao estiramento de carbonilas e carboxilas não conjugadas, típica de espectros de ligninas. 0 ombro localizado em $1650 \mathrm{~cm}^{-1}$ corresponde ao estiramento de carbonilas e carboxilas conjugadas, além de ligações $\mathrm{C}=\mathrm{C}$. A conjugação com uma ligação $\mathrm{C}=\mathrm{C}$ aumenta o deslocamento dos 
elétrons $\pi$ de ambos os grupos insaturados. No grupo $C=0$, a deslocalização dos elétrons $\pi$ reduz 0 caráter de ligação dupla e leva à absorção em menores números de onda.

Na região dos aromáticos, verificam-se bandas a 1600, 1500 e $1425 \mathrm{~cm}^{-1}$, relacionadas às vibrações do anel aromático e à deformação C-H combinada com a vibração do anel aromático a $1460 \mathrm{~cm}^{-1}$.

A Tabela 2 exibe os resultados da composição elementar do jornal e do resíduo da hidrólise, bem como do Poder Calorífico Superior calculado a partir da fórmula de Laut (VALE et al., 2000). Percebe-se que o teor de carbono do resíduo de hidrólise é bastante superior ao do jornal. Isso porque o jornal é composto por frações de celulose, hemicelulose e lignina e essas macromoléculas apresentam teores de carbono distintos entre si, sendo a lignina a que possui os maiores teores, da ordem de 60\% (FENGEL e WEGENER, 1989). A fração polissacarídica (celulose e hemicelulose) apresenta teores de 44,44 \% (glucana) e 45,45 \% (xilana).

Tabela 2 - Composição elementar e poder calorífico superior

\begin{tabular}{|c|c|c|c|c|c|c|}
\hline Material & $\% \mathrm{C}$ & $\% \mathrm{H}$ & $\% 0$ & $\% \mathbf{N}$ & $\% S$ & PCS* (J.g-1) \\
\hline Jornal & $46,81 \pm 0,18$ & $5,82 \pm 0,03$ & 44,97 & $1,56 \pm 0,19$ & $0,84 \pm 0,08$ & 17455 \\
\hline Resíduo da Hidrólise & $59,36 \pm 0,11$ & $5,42 \pm 0,03$ & 32,52 & $1,82 \pm 0,43$ & $0,88 \pm 0,14$ & 23078 \\
\hline
\end{tabular}

O poder calorífico dos materiais analisados apresentou diferenças bastante consideráveis, que estão associadas à composição dos materiais. Os polissacarídeos apresentam menores valores de poder calorífico em relação à lignina e, uma vez que o resíduo da hidrólise é composto basicamente por lignina, este foi o que apresentou os maiores valores.

Analisando o processo geral de hidrólise, recuperação do resíduo e tratamentos para destoxificação, considerando todos os fatores de diluição e rendimentos obtidos neste trabalho, verifica-se que, partindo-se de $1 \mathrm{~kg}$ de jornal, é possível produzir um hidrolisado contendo $341,3 \mathrm{~g}$ de açúcares fermentescíveis e ainda 195,6 g de resíduo sólido capaz de gerar 4,51 MJ de calor. Esse processo se encaixa no conceito de biorrefinaria, que trata do aproveitamento integral das frações de biomassa, gerando energia para o processo e açúcares fermentescíveis, agregando, dessa forma, valor ao material.

\section{CONCLUSÕES}

A partir do que foi exposto, é possível concluir que:

- 0 jornal é um material composto por cerca de $74 \%$ de polissacarídeos, $20 \%$ de lignina e $4 \%$ de cinzas inorgânicas;

- É possível produzir, por meio de hidrólise ácida do jornal, um hidrolisado rico em açúcares fermentescíveis;

- O resíduo da hidrólise é composto basicamente por lignina e uma fração de polissacarídeos não

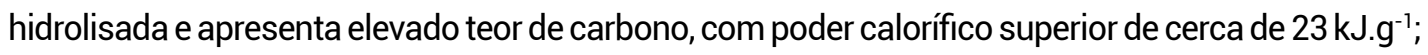

- No processo estudado, a partir de $1 \mathrm{~kg}$ de jornal, podem ser produzidos $341,3 \mathrm{~g}$ de açúcares fermentescíveis e 4,51 MJ de energia. 


\section{REFERÊNCIAS}

BRACELPA. Associação Brasileira de Celulose e Papel. Relatório Estatístico. Disponível em: <bracelpa. org.br>. Acesso em: 29 nov. 2014.

EK, M.; GELLERSTEDT, G.; HENRIKSSON, G. Pulp and Paper Chemistry and Technology. Berlin: Walter de Gruyter, v.3, 2009.

FENGEL, D.; WEGENER, G. Wood: Chemistry, Ultrastructure, Reactions. Berlin: Walter de Gruyter, 1989.

GAMBARATO, B. C. Isolamento e caracterização de ligninas de palha de cana-de-açúcar. 2014. Tese (Doutorado em Ciências). Escola de Engenharia de Lorena - USP. Lorena, 2014.

HON, D. N. S.; SHIRAISHI, N. Wood and cellulosic chemistry. 2. ed. New York: Marcel Dekker, 2001.

MAZIERO, P. Estudos topoquímicos durante obtenção de etanol a partir de celulose de bagaço e palha de cana-de-açúcar. 2013. 171 f. Tese (Doutorado em Ciências). Escola de Engenharia de Lorena - USP, Lorena, 2013.

ÖHREN, K. et al. Effect of hemicellulose and lignin removal on enzymatic hydrolysis of steam pretreated corn stover. Bioresource Technology, v. 98, n. 13, p. 2503-2510, 2007.

RAMOS, L. P. The chemistry involved in the steam treatment of lignocellulosic materials. Química Nova, v. 26, n. 6, p. $863-871,2003$.

VALE, A. T. et al. A. Energy production of stem of Eucalyptus grandis Hill ex maiden and Acacia mangium Willd in different levels of fertilization. CERNE, v. 6, n. 1, p. 83-88, 2000.

WU, F.; HUANG, S.; SHIH, I. Sequential hydrolysis of waste newspaper and bioethanol production from the hydrolysate. Bioresource Technology, v. 167, p. 159-168, 2014.

$\mathrm{XU}$, F. et al. Comparative study of organosolv lignins from wheat straw. Industrial Crops and Products, v. 23, p. $180-193,2006$. 